\title{
Compliance assessed by the Medication Event Monitoring System
}

\author{
N F Olivieri, D Matsui, C Hermann, G Koren
}

\begin{abstract}
The accurate assessment of patient compliance is especially crucial in evaluating the efficacy of a new treatment. Because of the problems associated with parenteral desferrioxamine, the development of a safe, effective, and convenient iron chelator is of high priority. The high morbidity and mortality associated with iron overload requires careful evaluation of the ability of any new agent to promote long term effective iron chelation. Patients' compliance with an orally available chelating agent, 1,2,-dimethyl-3-hydroxypyrid4-one (L1), that has been demonstrated to induce in vivo iron excretion equivalent to that of desferrioxamine during supervised short term administration, was examined. Compliance was assessed in seven patients by patient inverview, by daily diaries reviewed monthly with each patient, and with the use of the Medication Event Monitoring System (MEMS) standard pill bottles with microprocessors in the cap that record the timing and frequency of bottle openings. L1 was dispensed in MEMS containers to the patients, who, unaware of their significance, recorded compliance using a daily diary. Overall compliance rate (\% of prescribed doses taken) measured by MEMS was 88.7 $\pm 6.8 \%$. When 'doubling of doses' was accounted for, significantly poorer compliance with L1 was noted by MEMS $(91 \cdot 7 \pm 7 \cdot 4 \%)$ than by patients' diaries $(95 \cdot 7 \pm 5 \cdot 2 \%)$. There was no significant difference in patient compliance recorded between the first and last 30 day period of drug administration. MEMS can eliminate the confounding variable of erratic patient compliance in the evaluation of a new drug's efficacy. As MEMS cannot distinguish a missed dose from one doubled at the next bottle opening, the use of patient diaries is a useful adjunct to the accurate assessment of compliance and should be combined with the use of MEMS.
\end{abstract}

Evaluation of the therapeutic efficacy of a treatment regimen may be confounded by erratic patient compliance, the accurate assessment of which is especially crucial in demonstrating the benefit of any new treatment. The Medication Event Monitoring System (MEMS, Aprex Corporation, Fremont, California) standard pill containers with microprocessors in the cap, to record the timing and frequency of bottle openings, are a useful adjunct to estimates of compliance using patients' self reporting and/or pill counts. ${ }^{1}$ Recently, we reported effective iron chelation with supervised short term administration of oral 1,2-dimethyl-3hydroxypyrid-4-one ( $\mathrm{Ll}$ ) in iron loaded patients poorly compliant with parenteral desferrioxamine. ${ }^{2}$ Because of the high morbidity and mortality associated with tissue iron overload, ${ }^{3}$ the ability of $\mathrm{Ll}$ to promote long term effective iron chelation in these pateints must be carefully evaluated. However, its therapeutic benefit may be negatively influenced by poor compliance in patients with a history of not taking their desferrioxamine as instructed.

\section{Patients and methods}

PATIENTS

Seven patients with transfusion dependent homozygous $\beta$ thalassaemia began iron chelating treatment with oral L1. Four patients were started on L1 because of erratic compliance with nightly subcutaneous desferrioxamine treatment and three patients because of severe desferrioxamine neurotoxicity. ${ }^{4}$ The mean (range) age of the patients was $17 \cdot 4(10-22)$ years. In five patients drug administration was self supervised; two sisters, age 10 and 12 years, were supervised by a parent. Before Ll treatment the patients had administered nightly subcutaneous desferrioxamine, with varying degrees of compliance, for a mean (range) period of $10.6(8-12)$ years.

All patients, and the parents of the two children under 16 years, were aware of the importance of compliance with chelation treatment in the prevention of fatal iron related organ dysfunction. Written informed consent was obtained from each patient or parent at the time of initiation of $\mathrm{Ll}$ treatment. The experimental aspects of the drug administration and the lack of any alternative method of preventing and treating iron overload was emphasised. The nature of MEMS was unknown to the patients or parents, who also recorded compliance using a daily diary, reviewed on a monthly basis by one investigator. Patients were instructed to return all pill containers at their monthly visit at which time the remaining pills were counted.

Approval for the study was obtained from the Hospital for Sick Children human subjects' review committee.

\section{METHODS}

L1 capsules were dispensed in MEMS containers, which are standard size pill bottles fitted with a cap that contains a microprocessor. ${ }^{1}$ Each bottle opening and closing was recorded as a presumptive dose. Data were retrieved 
monthly by connecting the container to a microcomputer communication port. Each battery operated cap collected up to 350 events between visits for data retrieval. Collected data were sent on a diskette to the Aprex Corporation for analysis. Information was provided as listings of the date and time of individual bottle openings and closings, the duration of opening, and the hours elapsed since the previous dose. Also provided were the ranges of dose intervals and the times doses were taken.

DOSAGE

Each patient was instructed to take $\mathrm{Ll}$ at eight hour intervals, and to supplement any missed dose at the time of the day's subsequent dose. Thus, if a patient doubled any dose, only one bottle opening was recorded. To adjust the dose to $75 \mathrm{mg} / \mathrm{kg} /$ day, five patients administered L1 at two capsule strengths $(300 \mathrm{mg}$ and $600 \mathrm{mg}$ capsules) at different dosing frequencies; two patients administered one capsule strength only (300 mg capsules). For example, one patient administered two $600 \mathrm{mg}$ capsules three times daily, and one $300 \mathrm{mg}$ capsule one time daily; both regimens were recorded by diary and the MEMS device. Dosing patterns therefore included three times a day in four patients, and three times a day plus once daily in three patients.

\section{ASSESSMENT OF COMPLIANCE}

For the purposes of this analysis, only the omission of doses was considered as true noncompliance with L1 treatment. Conversely, the doubling of a missed dose of $\mathrm{Ll}$ at the time of the day's subsequent dose, as instructed, was not considered as non-compliance. The number of days during which delays of over 60 minutes in drug administration was also recorded. As failure to comply with the once daily regimen was likely to be reflected in the three times a day regimen, only the latter was included in the calculations of compliance.

\section{STATISTICAL METHODS}

Data are presented as mean (SD). Mean values were compared by paired Student's $t$ test as appropriate.

\section{Results}

The compliance rate, calculated as the percentage of prescribed doses taken, as estimated by MEMS, was $88 \cdot 7(6 \cdot 8) \%$. When allowance was made for supplementation of a missed dose at the time of the subsequent dose, a compliance with $\mathrm{Ll}$ increased to $91 \cdot 7(7 \cdot 4) \%$. The corresponding figures for percentage of days that the correct number of doses were taken were 71.6 $(15 \cdot 1) \%$ and $79 \cdot 9(18 \cdot 2) \%$ respectively.

Despite the importance of taking their medication at regular intervals having been explained, patients often delayed taking their $\mathrm{Ll}$, with delays greater than 60 minutes occurring more than half of the time $(55 \cdot 6(25 \cdot 8) \%$ of total days observed).

Patients' diaries reported a compliance rate of $95 \cdot 7(5 \cdot 2) \%$ of prescribed doses taken. This form of self monitoring lead to a significantly greater estimation of compliance compared with MEMS which yielded a corresponding value of $91 \cdot 7(7 \cdot 4) \%(p=0 \cdot 039)$.

Compliance was also assessed by pill counts. (calculated as [number of missing pills/number of pills prescribed for the interval] $\times 100 \%$ ). $N \Phi$ differences were noted in the evaluation of compliance by pill counts $(95.7(4 \cdot 8) \%)$ as compared with that by MEMS $(91 \cdot 7(7 \cdot 4) \%)$.

Overall, compliance with treatment was nof significantly different during the first month of Ll treatment when compared with the las (fourth) month, when missed doses werę considered.

\section{Discussion}

Because of the difficulties associated witly administration of nightly subcutaneous desfer? rioxamine for the treatment of iron overload, ${ }^{4}$ \& convenient and non-irritating substitute fo desferrioxamine, in the form of an orally avail $\vec{\omega}$ able iron chelating agent, has been sought fore many years. Until recently this search has been unsuccessful. However, recently we $\mathrm{w}^{2}$ and others ${ }^{5}$ have demonstrated the short term efficacy of $L \vec{b}$ in patients with iron overload. This treatmenth cannot be extended to all patients with irors overload until its long term efficacy and safety are demonstrated.

Presently, iron excretion in a cohort ofo patients with poor or erratic compliance with desferrioxamine is being evaluated. In general demonstration of efficacy of any new agent is confounded when patients do not adhere to prescribed regimens. ${ }^{6}$ In this particular instanceू the potential benefits of $\mathrm{Ll}$ must be evaluated in a cohort of patients whose compliance with treatment has already been demonstrated to bes erratic. We have reported a significant decline? in compliance with a regimen of nightly sub? cutaneous desferrioxamine between the ages of 10 and 20 years, frequently resulting in fata iron accumulation. ${ }^{7}$ Because of the high morbidity and mortality associated with tissue iron overload, ${ }^{3}$ the ability of $\mathrm{Ll}$ or any new agent to promote long term effective iron chelation must be carefully evaluated.

In the evaluation of patient compliance, threeo different measures have been utilised: self reports, collateral reports, and objectiveo measures. ${ }^{8}$ The inaccuracies in self reports off health related behaviour have been extensivelyn reviewed. ${ }^{9}$ Self reports are plagued by failure ofo memory, misunderstanding of treatment recommendations, and differences in internal standards of evaluations of behaviour. ${ }^{8}$ Purpose $-?$ ful omission of the truth to avoid recrimination, 0 embarrassment, or rejection by the prescribing physician $^{10}$ is also a major contributor to errors@ in self reporting of compliance. ${ }^{11} 12$

Collateral reports of compliance include reports of physicians, nurses, other healtho professionals, friends, and family members? The accuracy of collateral reports is a function of familiarity with the patients. ${ }^{13}$ In general, medical practitioners' ratings of their patients' compliance tend to be grossly inaccurate; 
physicians have been shown to routinely overestimate their patients' compliance and to be unable to distinguish compliant and noncompliant patients. 14

Objective measures of compliance include pill counts and urine or blood assay of the drug or its metabolites. Pill counts can be confounded if unused bottles are mislaid or deliberately not returned. The taking of occasional pills can balance with days of missed pills to provide an erroneous impression of adherence. ${ }^{15}$ Serum or urine concentrations of the drug are most useful for compounds with long half lives because results provide an approximation of use during the preceding week or longer. For rapidly cleared drugs, brief intake before the test can result in adequate concentration, erroneously suggesting regular medication use. ${ }^{1}$

The ideal standard of compliance measurement should be simultaneously unobtrusive (to avoid patient sensitisation and maximise cooperation), objective (to produce discrete and reproducible data for each subject), and practical (to maximise portability and minimise cost). ${ }^{16}$ MEMS, a novel method used in our study, can measure adherence to a treatment regimen on a daily and hourly basis and is minimally intrusive to the ambulatory outpatient. MEMS has been reported as useful to study compliance with prescribed regimens and to compare results with other traditional measures.

In the present study, we have used MEMS to evaluate compliance with a new treatment in patients, of whom some were known to be previously poorly compliant with a regimen of nightly subscutaneous desferrioxamine. As previously noted, compliance is reduced when the treatment regimen is complex, of long duration, dependent on an alteration of the patient's lifestyle, inconvenient, or expensive. ${ }^{17-20}$ Frequency of dosing in one study was a more important factor for compliance than the number of different medications taken at each dosing interval. ${ }^{21}$ Frequent (three to four times daily) dosing, a requirement with $\mathrm{Ll}$, would therefore be expected to increase inconvenience and diminish compliance.

In the present study, patients' diaries reported a compliance rate higher than that recorded with MEMS. In contrast, for the same regimen, compliance as measured by MEMS was significantly less at $91 \cdot 7(7 \cdot 4) \%$ of doses taken. Our findings of consistent over reporting of compliance confirms previous suspicions that, in the absence of objective measures of compliance, ${ }^{11}$ patients will exaggerate the degree of their compliance with a treatment regimen, perhaps to gain acceptance, support, or approval from the prescribing physician. ${ }^{12}$ The use of patients' diaries does eliminate to a degree the confounders of memory failures in compliance reporting. ${ }^{6}$ In addition, because patients were instructed to double any missed dose of $\mathrm{Ll}$ at the subsequent one, the adjunctive use of patients' diaries was necessary to distinguish lack of compliance with a dose of $\mathrm{Ll}$ from one doubled at the next presumptive dose while using MEMS.

Estimation of compliance by pill counts $(95 \cdot 7$ $(4 \cdot 8) \%$ ) was identical to MEMS. With the continuation of this study the role of pill counts will need to be further examined to determine if, over time, they will provide a satisfactory alternative to the more expensive MEMS monitoring.

Overall, compliance with treatment was not significantly different in the first month of $\mathrm{Ll}$ treatment when this was compared with the last month when missed doses were considered. The importance of the duration of a regimen in compliance with treatment is highlighted by a study that found a $30 \%$ incidence rate of dosage errors in patients with diabetes for one to five years, but an $80 \%$ dosage error rate in patients who had the disease for over 20 years. ${ }^{22}$

Despite its obvious advantages, MEMS still does not provide a foolproof method of compliance assessment. In the same way that it must be assumed that a bottle opening means the drug was ingested, it must be assumed that the absence of a bottle opening means the dose was not taken. These conditions, however, may not always hold true. For example, patients not wishing to carry their pill bottles with them may remove several doses at one time (thus registering only one bottle opening), yet still take their medication according to their prescribed schedule. This scenario is most likely to occur when the patients are unaware that their compliance is being monitored and are unaware of the nature of the MEMS container, as in the present study.

Our study focused on patients' compliance. However, generally drug trials are analysed by 'intention to treat', as compliance or lack of compliance is part of the assessment of the usefulness of a drug in clinical practice. However, even in the larger context of 'intention to treat', accurate analysis of patients' compliance is crucial.

Our study demonstrates that MEMS is a useful aid in quantifying patients' compliance during the evaluation of the therapeutic efficacy of a new drug. In the evalution of a new drug it is crucial to establish appropriate compliance before one can reject the agent as ineffective. As MEMS cannot distinguish a missed dose from one taken (that is doubled) at the next bottle opening, the use of patients' diaries should be a useful adjunct to this method of assessment of compliance.

Supported by a grant (MA-\#10601) from the Medical Research Council of Canada. NFO and GK are career scientists of the Ontario Ministry of Health.

1 Cramer JA, Mattson RH, Prevey ML, et al. How often is medication taken as prescribed? A novel assessment technique. FAMA 1989;261:3273-7.

2 Olivieri NF, Koren G, Hermann C, et al. Comparison of ora iron chelator $\mathrm{Ll}$ and desferrioxamine in iron-loaded iron chelator $L 1$ and desferrioxa

3 Cohen A. Iron overload in the pediatric patient. In: Oski FA ed. Pediatric hematology. Hematology/oncology clinics of ed. Pediatric hematology. Hematology/oncology clinics of

4 Olivieri NF, Buncic JR, Chew E, et al. Audiotory and visual neurotoxicity in patients receiving subcutaneous deferoxamine infusions. $N$ Engl f Med 1986;314:869-73.

5 Kontoghiorghes GK. Effective chelation of iron in $\beta$ thalassemia with the oral chealtor L1. BMF 1987;295:1509-12.

6 Eraker SA, Kirscht JP, Becker MH. Understanding and improving patient compliance. Ann Intern Med 1984;100 258-68.

7 McGee A, Liu P, Benson L, Koren G, Olivieri N. Cardiac disease free survival in thalassemia major patients receiving deferoxamine: an update on the Toronto cohort. Bloo 1989;74(suppl 1):311a. 
8 Hays RD, DiMatteo MR. Patient compliance assessment. Fournal of Compliance in Health Care 1981;2:37-53.

9 Cannell CF, Oksenberg L, Converse JM, eds. Experiments in interviewing techniques: field experiments in health reporting, 1971-77. Ann Arbor, Michigan: Survey Research Centre Institute for Social Research, 1977.

10 Hippocrates. On decorum and the physician. London WH, ed. Jones WHS, translator, 1923.

11 Crowne DP, Marlowe D. A new scale of social desirability independent of psychopathology. Fournal of Consulting Psychology 1960;24:349-54.

12 Gordis L, Markowitz M, Lilienfield AM. The inaccuracy in using interviews to estimate patient reliability in taking using interviews to estimate patient reliability
medications at home. Med Care 1969;7:49-54.

13 Witenberg SH, Blanchard EB, McCoy G, Suls J, McGoldrick MD. Evaluation of compliance in home and center hemodialysis patients. Health Psychol 1983;2:227-37.

14 Caron HS, Roth HP. Patients' cooperation with a medical regimen. $\mathcal{F A M A}$ 1968;203:922-6.
15 Norell SE. Improving medication compliance: a randomized clinical trial. $B M \mathcal{F}$ 1979;ii:1031-3.

16 Rudd P. In search of the gold standard for compliance measurement. Arch Intern Med 1979;139:627-8.

17 Haynes RB. A critical review of the 'determinants' of patients compliance with therapeutic regimens. In: Sackett DL, compliance with therapeutic regimens. In: Sackett DL, Haynes RB, eds. Compliance with therapeutic regimens.

8 Becker MH, Maiman KL M. Mrug therapeutics 1982. Comcepts for physians. New KL ed. Drug therapeutics 1982. Concepts for physicians. New

19 Johannsen WJ, Hellmuth GA, Sorauf T. On accepting medical recommendations. Arch Environ Health 1966;12: 63-9.

20 Matthews D, Hingson R. Improving patients' compliance: a guide for physicians. Med Clin North Am 1977;61:879-89.

21 Porter AM. Drug defaulting in a general practice. BMF 1969;i:218-22.

22 Charney E. Patient-doctor communication. Pediatr Clin North Am 1972;19:263-79.

Can cleaning carpets cause Kawasaki's curious clinical conglomerate? Colorado case-control comparison corroborates contentious connection

It stands to reason, doesn't it, that a disease like Kawasaki disease can't be due to something as simple as carpet cleaning; it's as silly as saying that something like pink disease could have been caused by teething powers or Reye's syndrome associated with giving aspirin. The suggestion about Kawasaki disease (I shall continue to call it a disease because it trips more lightly off the tongue, though I recognise the case for calling it a syndrome) was first made in Colorado in $1982^{1}$ but several subsequent studies failed to confirm the association. Now workers in Denver have resurrected the idea (Alan M Rauch and colleagues, Pediatrics 1991;87:663-9).

The largest epidemic of Kawasaki disease yet reported in the United States occurred in an area to the east of the Rocky mountains centred on Denver between October 1984 and January 1985. There were 62 cases in all, of whom 52 lived in the Denver metropolitan area. The case-control study included 26 patients. Controls matched for date and place of birth, race, and sex were selected at random from birth certificate records and a questionnaire was administered by telephone to the parents of patients and controls. The age range of the children was 3 months to 12 years with nearly $90 \%$ younger than 5 years. The incidence was significantly higher in blacks than in whites and highest of all in Asians (origin unstated). Carpet cleaning must be a popular activity in Denver because $62 \%$ of the patients and $20 \%$ of controls lived in homes where rugs or carpets had been cleaned within 30 days of the onset of the illness $(p<0.01$, odds ratio $5 \cdot 0,99 \%$ confidence interval $1 \cdot 1$ to $23 \cdot 1$ ) though the study rules stated that the area cleaned might be as small as 1 foot in diameter and the cleaning fluid might be a commercial cleaner or soap and water. Data from 35 patients in three studies suggested exposure to carpet cleaning in the period from 13 to 30 days before the onset of the illness in 33 and 0 to 12 days in two $(p=0.00005)$.

Other associations were looked for. It has been suggested that living near a body of water might predispose to the disease. In this study the median distance from a body of water (ditch, creek, or pond) for patients was 200 yards and for controls 500 yards $(p=0.06)$. No significant differences were found for antecedent illness in the child, illness in the family, parental income, and a list of 18 other factors.

It's odd, isn't it? Could the results be explained by recall bias? If so, why the clustering in the period between 13 and 30 days before the onset of the illness rather than more recently? There seems to be no chemical or toxic explanation. The authors suggest that an infectious agent might be stirred up by the cleaning and agitation of the carpets. The possible association with living near water could also suggest a small animal reservoir or an insect vector.

These studies must be telling us something, but what? 